\title{
The Orientation of Pre-Romanesque Churches in the Iberian Peninsula
}

\author{
Antonio César González-García • \\ Juan Antonio Belmonte
}

Published online: 8 January 2015

(C) Kim Williams Books, Turin 2014

\begin{abstract}
The orientation of Christian churches is a distinctive feature of its architecture, repeating patterns from early Christian times that show a general tendency to orientate their apses in the solar range, with a predilection for orientations near the astronomical equinox. We measured the orientation of a total of 167 churches built prior to A.D. 1086. A comprehensive statistical analysis of the sample indicates a decisive orientation pattern, with a clear tendency to orientate according to the standard tradition, albeit with certain particularities. Three subsamples are examined to find the tendencies at each different historical time. This exercise indicates that the main group of orientations seem to relate to the Canonical equinox on 25 March, while popular 'expected' orientations, such as that of the rising sun on the day of the patron saint of the church, are completely absent. Other groups of orientations are specific to each period, such as that towards sunrise on Saint James's day, important only after the discovery of the saint's tomb in the ninth century.
\end{abstract}

Keywords Pre-Romanesque architecture - Church orientation · Christian religion · Iberian Peninsula · Archaeostronomy · Julian calendar · Gregorian calendar · Mozarabic architecture

A. C. González-García ( $₫)$

Instituto de Ciencias del Patrimonio Incipit CSIC, Santiago de Compostela, Spain e-mail: cesar.gonzalez-garcia@incipit.csic.es

\section{J. A. Belmonte}

Instituto de Astrofísica de Canarias La Laguna, Departamento de Astrofísica, Universidad de La Laguna, Tenerife, Spain

e-mail: jba@iac.es 


\section{Introduction}

The study of the arrangement of Christian churches has been of interest since time immemorial and this key factor, so representative of their architecture, has recently resulted in an avalanche of new studies in the literature. According to the texts of early Christian writers and apologists, churches should lie along a particular orientation, that is, the priest had to stand facing east during services. Origen recognized this, as well as Clement of Alexandria and Tertullian, and it was finally made official at the Council of Nicaea of 325. Saint Athanasius of Alexandria, also in the fourth century, says that the priest and participants must face eastward, where Christ, the Sun of Justice, would shine at the end of time [eccleasirum situs plerumque talis erat, ut fideles facie altare versa orientem solem, symbolum Christi qui est sol iustitia et lux mundi interentur (...)] for a thorough analysis of the early sources and methods of orientation see Vogel (1962).

At first, not all early Christian basilicas were built with the apse or the head of the church facing east. In this respect, Delgado-Gómez (2006) indicates that, of the twenty Christian basilicas built during the time of Constantine and his successors in Rome, Jerusalem, Constantinople and the north of Africa, eighteen are located approximately in the east-west line, eleven of which have the apse directed towards the west. However, it is interesting to note that in those cases the priest is facing east, because the altar is located between him and the congregation.

Between the third and seventh centuries the Apostolic Constitutions (II, 7) clearly indicate that churches should be built facing east. In the fifth century, Sidonius Apollinaris and Paulinus of Nola indicate that the apse must look to the east, towards the equinox, a view later confirmed by the Pope Virgilio and Isidore of Seville in his Etymologiae (XV, 4; McCluskey 1998). Honorio Augustodunensis confirmed this during the Middle Ages (eleventh-twelfth centuries; Gemma animae I, 129 de situ ecclesiae):... ecclesiae ad orietem vertuntur ubi sol oritur..., as well as other authors such as William Durand (twelfth-thirteenth centuries; Rationale divinorum officiorum, V, 2, 57):... versus orientem, hoc est, versus Ortum aequinoctialem solis, nec vero aestivale Solstitium..., which clearly indicates the direction to follow, the equinox, avoiding the solstice.

This eastern orientation has a clear symbolism as the direction in which the sun rises and therefore represents Christ as the Sun of Righteousness, who will arise from there on Judgment day. It was also roughly the area towards which the Christians in the west would be facing Jerusalem (McCluskey 2004, 2010).

However, these indications still remain somewhat ambiguous regarding how to build the church pointing to the east. The sources point toward the need to orientate the apse to equinoctial east, but one may ask to which equinox should the apse be facing. As mentioned by McCluskey (2004), there are several possibilities to obtain an equinox: the Roman vernal equinox occurred on 25 March, while the Greek occurred on 21 March, as was reflected in the Council of Nicaea. But we may use other definitions, such as the entry of the sun in the sign of Aries or the autumnal equinox (24 or 21 September for the traditional Roman or Greek equinoxes). Each of these definitions (Ruggles 1999; González-García and Belmonte 2006) would result in different dates, and therefore in slightly different orientations. Another 
important point to consider is the use of the Julian calendar in the Middle Ages and much of the Renaissance. If we concentrate on a particular cultural definition of Equinox (i.e., on a particular date, such as 25 March), the nature of the Julian calendar would make such a date shift with respect to the seasons, a fact that would be reflected in a systematic change in orientation.

It is interesting to note that later sources point to other possibilities for obtaining the church orientation such as the use of sunrise on the day of the patron saint of the church, the position of the sun on the day that construction of the church started, or on some specific important festival for the community. For instance Johnson, although mentioning the idea of orientating the churches 'to the point of sunrise which corresponded to the day of the patron saint to whom the church was dedicated' (Johnson 1912, p. 225), also indicates that this idea is not traceable further back than to the mid-seventeenth century, in a manuscript where Silas Taylor says: '...I have experimented (sic) some churches and have found the line to point to that part of the horizon where the sun arises on the day of the Saint to whom the church is dedicated' (Johnson 1912, p. 225). Something similar is described by the British poet William Wordsworth (1888; "On seeing the Foundation preparing for the erection of Rydal Chapel, Westmoreland") in a poem written in 1824 but published several years later on the occasion of the foundation of Rydal Chapel:

He [the sun] rose, and straight-as by divine command

They, who had waited for that sign to trace

Their work's foundation, gave with careful hand

To the high Altar its determined place;

Chauncey, however, states, 'One end of every Church doth point to such a place where the sun did rise at the time the foundation thereof was laid... and by the standing of these churches, it is known at what time of the year the foundations of them were laid' (Chauncey 1700, p. 88). This does not tell us, however, the time of year when construction started. It is important to note that all these accounts are rather late in the history of church building.

The study of the orientation of European medieval churches, along with the pyramids of Egypt and European megaliths, is one of the longer-standing subjects worked on in archaeoastronomy (Nissen 1906). The first author of this present paper has recently carried out a review of the work in this field (González-García 2014), which shows that the requirements for the eastward orientation is followed fairly consistently throughout Europe, at least during the Middle Ages, as shown in Fig. 1 (graph a). All areas considered in his study follow this pattern, with a clear predominant peak centered on the equinox, noting that, in many cases, especially in Western Europe, this maximum is slightly shifted northward relative to the astronomical value, perhaps indicating a use of specific dates for the equinox (perhaps 25 March).

However, a constant in the literature on the orientation of the churches is that they are orientated towards sunrise on the anniversary of the patron saint, but in early writings and well into the Middle Ages there is no epigraphic endorsement or archaeological evidence for such a claim. We find no such trend until the emergence of religious orders in late medieval times. González-García (2014) indicates that in 

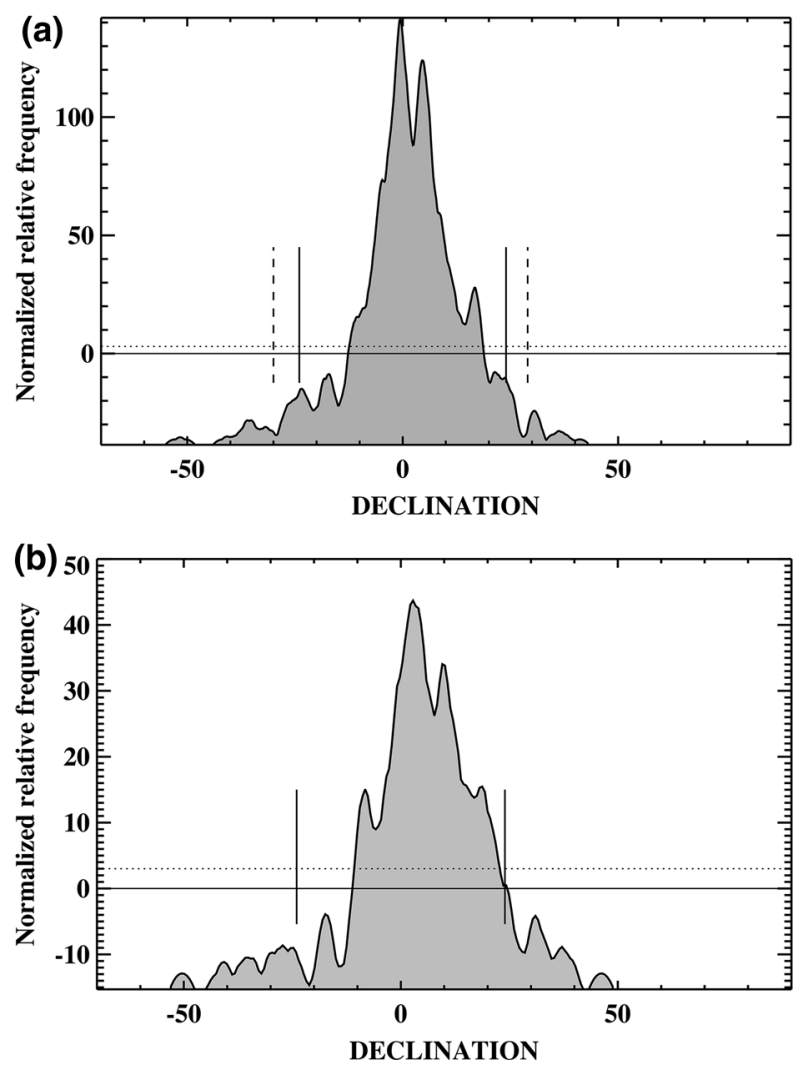

Fig. 1 Declination histograms for European and Iberian churches. Graph a shows the data for more than 560 medieval churches from Europe. Vertical solid lines indicate the extreme solar range (between $23.5^{\circ}$ and $23.5^{\circ}$ ), while the vertical dashed lines indicate the lunar extremes. The astronomical equinox has a $0^{\circ}$ declination. This figure indicates that most churches are orientated almost towards the east, with avoidance at the solstices. Graph b shows the histogram of declinations for the 167 measurements of the Iberian Peninsula presented in this paper. The largest concentration of orientations is slightly north of the astronomical equinox. Secondary maxima appear at $\sim 10^{\circ},-10^{\circ}$ and $20^{\circ}$. These could be related to some of the major Christian festivities celebrated in the region. See text for further details

areas of Germany, and perhaps in England and France, there might be a concern for certain saints in some sites, but these buildings are generally Romanesque monastic churches and Gothic cathedrals, and therefore later than the churches we are interested in. An interesting and well-documented case occurs in Slovenia, where Caval (2009) found evidence for a particular predilection for the feast called the Chair of St. Peter. Such an inclination is reflected in the orientation of a significant number of churches in the direction of sunrise that day. McCluskey (2004) indicates that something similar happens in England for the orientation of Romanesque churches, where shrines with Marian devotions might follow this rule in a manner complementary to the eastward orientation. Interestingly, an exception to the rule of orientations towards the east occurs in North Africa, where churches were also constructed in opposite directions. There are a number of churches with orientations 
towards the west, a usual custom in early Christianity, as noted above (Esteban et al. 2001; Belmonte et al. 2007). González-García (2014) has also shown that most of the churches are aligned within the solar range, with concentrations at the equinoxes and solstices, which could provide clues about the process of Christianization in this region.

In the Iberian Peninsula and in the two archipelagos (the Balearics and the Canaries) the question of the orientation of churches has been researched in very general terms, although there are reports of particular illumination events within Romanesque churches at special moments, such as the Equinox (such as the phenomena occurring at Santa Marta de Tera and San Juan de Ortega, in the provinces of Zamora and Burgos respectively). This has led to unfounded claims regarding the possible cause of the deviation of some churches with respect to the standard orientation (see, for example, Godoy Fernandez 2004). It is of particular note that Pérez-Valcarcel (1998) has investigated the orientation of 187 Romanesque churches of the Camino de Santiago, but his data do not include the measurement of the angular height of the horizon, an unfortunately very common occurrence in many European studies. However, what does seem clear is that a general relationship between the orientation of these churches and sunrise on the date of invocation of the patron saint of the church has not been firmly established.

Our team has decided to launch a large-scale project in the Iberian Peninsula, the Balearic and Canary Islands. González-García and Belmonte (2014a) devoted particular attention to the period of the Asturian churches and their interaction with the dominant Muslim power in the south of the peninsula. Specifically, they found that the thirteen churches still existing from that period possess a canonical orientation, with the apse towards the eastern half of the horizon, although generally deflected several degrees north of east. Furthermore, the authors found that the mosques of Al-Andalus, whose qibla must have been facing Mecca and may thus have had orientations compatible with the canonical alignments of churches towards east, followed a peculiar pattern. On the one hand, a number of mosques were orientated towards southeast, while others follow the qibla of the Great Mosque in Cordoba to south-southeast, both provisions permitted by Islam under certain circumstances. Thus it would seem that Muslims in Al Andalus 'avoided' orientations that might confuse their temples with churches, while the Asturian shrines tend to avoid positions that mistake these temples with mosques (Fig. 2).

This is an excellent example of the interaction of religion, power and astronomy. Curiously, our team (see Gangui et al. 2015) has studied the orientations of the churches in Lanzarote from the period of the Castilian conquest in the fifteenth century until the nineteenth century, finding that a majority of churches follow the canonical orientation. However, a significant number had orientations dictated by the prosaic need to avoid the prevailing winds. Thus, we see that in exceptional circumstances, the canonical patterns can be altered. Finally, García Quintela (2014) have investigated the introduction of Christianity in the northwest of the Peninsula and the possible replacement of Indo-European (Celtic) cultic elements by Christians factors by introducing what they call a 'martyr landscape' through the orientation of the churches and the Christianization of their environments and the 


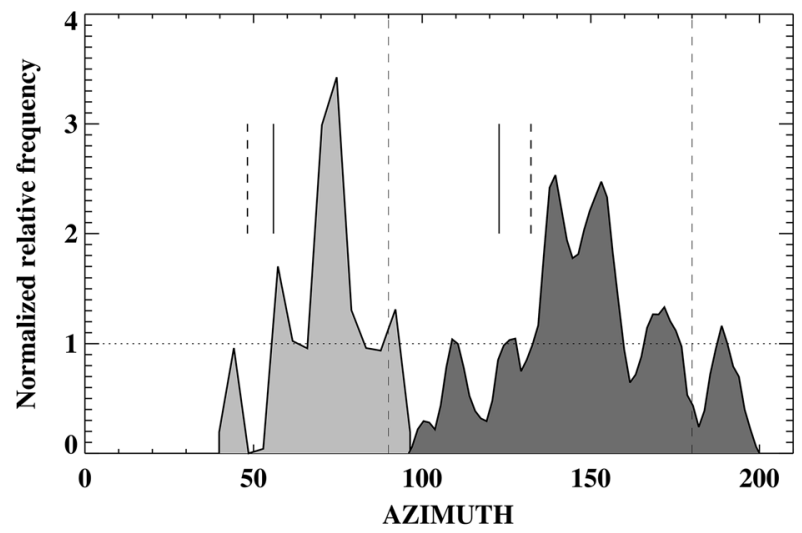

Fig. 2 Azimuth histogram forAsturian churches (light grey) and Andalusian mosques (dark grey). Both samples seem to be mutually exclusive. Adapted from González-García and Belmonte (2014a)

creation of myths and tales that channeled, modified, or replaced potentially pagan cults.

\section{The Church in Iberia in Pre-Romanesque Times}

From the fourth to the eleventh centuries we can distinguish four main phases marked by the Germanic and the Muslim invasions in the fifth and eighth centuries, respectively. Actually, A.D. 711 is a real point of inflexion in Iberian history. Prior to this date we can include the Palaeo-Christian and Visigoth periods, that is, from the late Christian Roman age and the settlement of certain Germanic kingdoms in the Peninsula, especially the Visigoth kingdom, to the decomposition of a world with the arrival of the Muslims. Then, after the reaction to the Arab invasion and the emergence of some centres of opposition in the northern part of the Peninsulaparticularly in Asturias - there is a period in which a number of churches with welldefined features were built within a very short time interval. The final period and architectural style (called Mozarabic for the Christian people escaping from Al Andalus to the liberated northern territories) corresponds to the first expansion of these new kingdoms to the south, until the conquest of Toledo in A.D. 1085 and the introduction by monks from France and Central Europe of the Romanesque style along the well-established path of the Camino de Santiago.

Christianity expanded steadily in the Peninsula during the final centuries of the Roman Empire, especially in urban areas and the Villae (Fig. 3).

In A.D. 411 a series of Germanic tribes entered the Peninsula, including especially the Suevi, in what is now Galicia and northern Portugal, and the Visigoths, who later settled in central and eastern Iberia. After a long period of wars, the Visigoths defeated and assimilated the Suevi and conquered the entire Peninsula to form a kingdom strong enough to build a large number of churches with special characteristics (Fig. 4). The church had great power within the Visigoth kingdom 

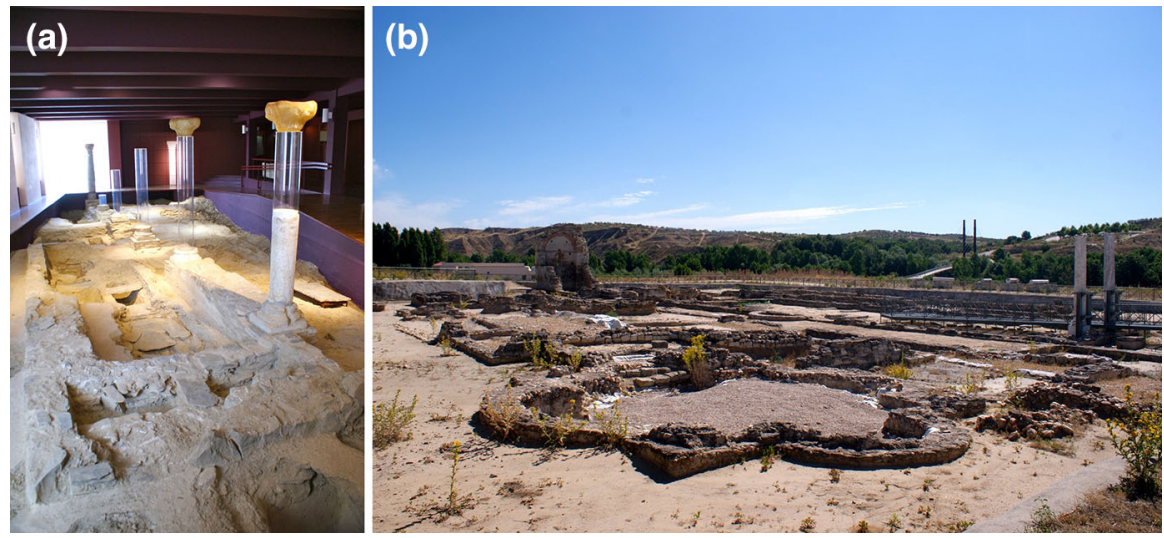

Fig. 3 Early Christian Churches in Hispania: a Palaeo-Christian basilica of Mertola (Portugal) and b the late Roman villa of Maternus, Carranque (Toledo). They date from the late Roman period (fourth century). Most Palaeo-Christian churches in Spain and Portugal have been found in archaeological excavations like these two examples

itself and became one of the pillars of the Visigoth state. The church participated in major decisions of civil life that were taken in the Councils. Important figures in this period were the aforementioned Saint Isidore and his brother, Saint Leander. The Visigoth churches had a very clear and relatively strict partitioning of their internal space, with a circulation within them during the celebrations where a rather complex ritual was performed (called Mozarabic for its later persistence under Arabic rule) that was different from that of the Roman Catholic Church and in some ways similar to the Orthodox church.

From A.D. 711 on, a series of Muslim armies from North Africa entered the Peninsula, conquering the entire Visigoth kingdom in just five years. A small number of territories in the north of the Visigoth kingdom (notably Asturias) did not completely submit to Muslim rule. In the period until the ninth century a fair number of churches were built in that kingdom. At present 15 of them are preserved in fairly good condition and have a number of formal characteristics that refer to the previous Visigoth period, such as the use of the horseshoe arch or the intricate internal compartmentalization.

In the late ninth century the capital of this kingdom was moved south, to León, in order to expand to more southerly regions. This epoch coincides with a period in which numerous groups of Christians from the Muslim-dominated south moved to the Christian kingdoms of the north for different causes, including the inability to build new churches or to restore old ones in the areas under Islamic rule. These Christians, called Mozarabic, built a large series of fascinating and eclectic churches in all these kingdoms, from Galicia to Catalonia, again with features that refer to the Visigoth kingdom, but with clear Muslim influences in the art (Figs. 5, 6).

A singular group of these churches are those built in the Pyrenees area integrated by the kingdom of Aragón (notably in the district of Serrablo), which show different characteristics, somehow more similar to the early Romanesque style. Interestingly, 

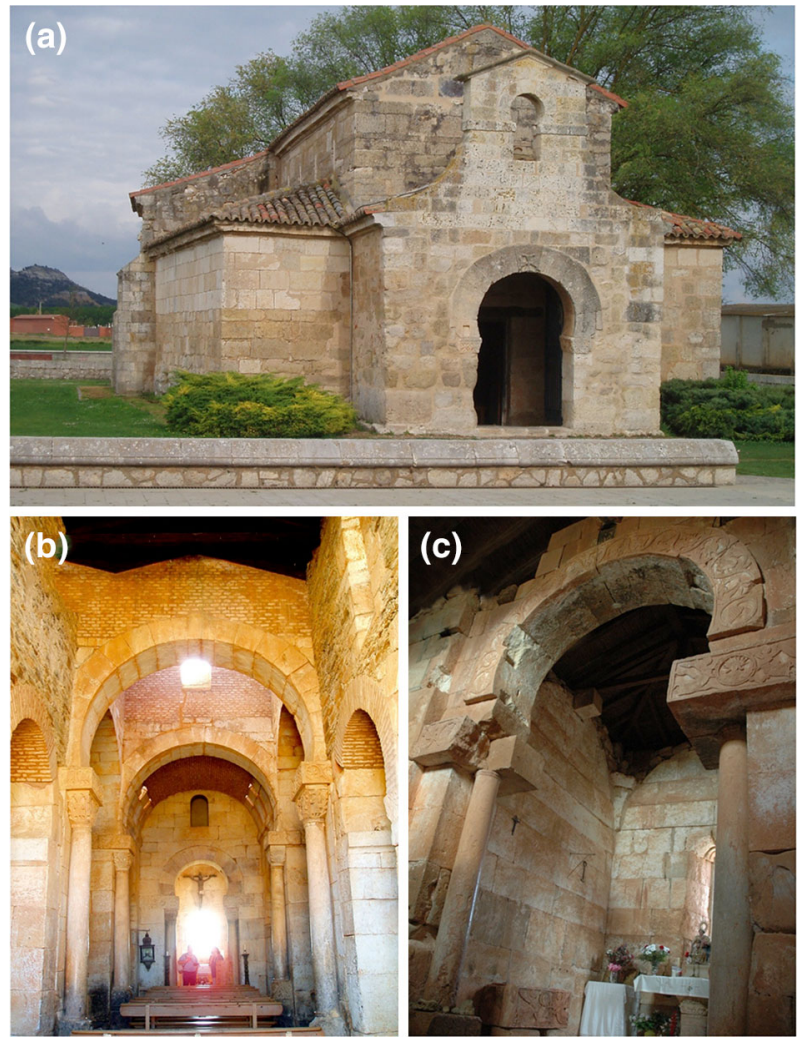

Fig. 4 Three examples of Visigothic churches. a The church of San Juan de Baños (Palencia), built by King Recceswinth c. A.D.661, b the interior of the church of San Pedro de la Nave (Zamora) and c Santa María de lasViñas (Burgos). Nave is one of the best-preserved examples of Visigoth architecture in Spain; however, it is not included in our measurements as it was moved from its original site nearly a century ago due to the construction of a water dam. The original site is now under water, and it was impossible to make measurements on site. Note in all cases the use of the horseshoe arch, that will later be characteristic of the Arab art of Al Andalus, and the lavish decoration of the capitals, with astronomical motifs in the case of Viñas

it is precisely at this time when, after the discovery of the tomb of the Apostle James in A.D. 834, the pilgrimage to Compostela along the Camino de Santiago was first promoted, opening the gate for the rise of the later Romanesque period.

\section{Data Sample and Statistical Analysis}

Given these accounts, we have undertaken a systematic survey on the orientation of the pre-Romanesque churches in Spain and Portugal. The number of these churches in Iberia is varied as it strongly depends on the state of preservation. There are large numbers of churches that have several different building phases, and previous stages are commonly reused. Our criterion to select those in our 

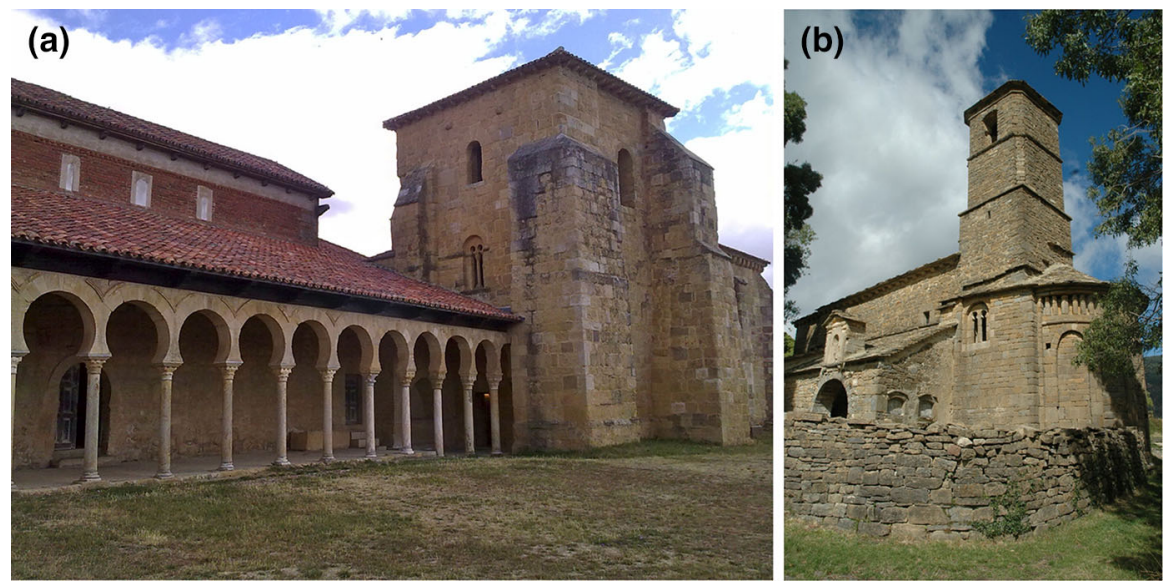

Fig. 5 Two examples of Mozarabic churches, a San Miguel de Escalada in León, and b Susin (Huesca), an example of a Serrables church. Note the use of the horseshoe arch architecture evoking Arabic roots, although the use of this type of arc is prior to the Muslim invasion of the peninsula. The Serrables style conjoins the Mozarabic and early-Romanesque characteristics in an eclectic way
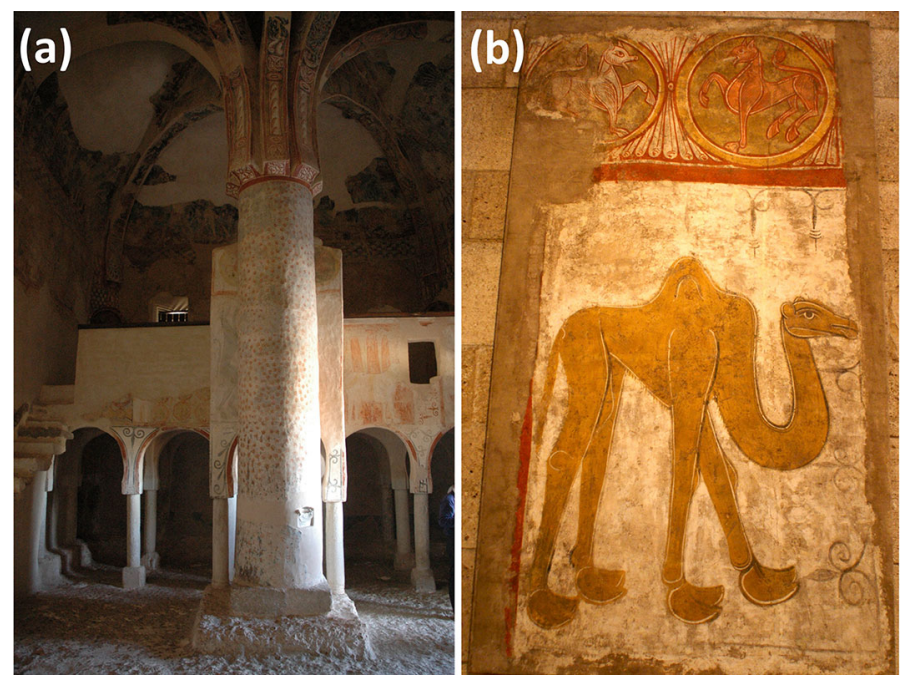

Fig. 6 San Baudelio de Berlanga (Soria). This small Mozarabic hermitage shows a lavishly decorated architecture including impressive features such as the central column in the shape of a palm tree (a). The church was completely covered by frescoes housing some of the most impressive paintings from this period (b)

sample was that at least the baseline of the church should be recognizably from a pre-Romanesque period. In several instances this is quite easy, as some have undergone comprehensive restorations in the recent past, although in others it is more difficult to discern. Some churches present several phases with changes of orientations. These are sometimes incorporated in a single building, showing a 
Table 1 Orientation of 167 pre-Romanesque churches in Spain and Portugal

\begin{tabular}{|c|c|c|c|c|c|c|c|c|c|}
\hline Place & Monument & Period & $\begin{array}{l}\varphi \\
(\% /)\end{array}$ & & $\begin{array}{l}\lambda \\
\left({ }^{\circ},\right)\end{array}$ & & a $\left({ }^{\circ}\right)$ & $\mathrm{h}\left({ }^{\circ}\right)$ & $\delta\left({ }^{\circ}\right)$ \\
\hline Veranés & Sta. María & Paleo. & 43 & 29 & 5 & 45 & $583 / 4$ & 0 & 21.68 \\
\hline Bóveda & Sta.Eulalia & Paleo. & 42 & 58 & 7 & 41 & $931 / 4$ & 0 & -2.77 \\
\hline Marialba & Martirium & Paleo. & 42 & 32 & 5 & 32 & 149 & 0 & -39.7 \\
\hline Augasantas & St.Marina & Paleo. & 42 & 14 & 7 & 47 & $1193 / 4$ & $61 / 2$ & -16.9 \\
\hline Ampurias & Ig. Neapolis & Paleo. & 42 & 8 & 3 & 7 & 75 & 0 & 10.67 \\
\hline Ampurias & Sta. Magdalena & Paleo. & 42 & 7 & 3 & 6 & 53 & $71 / 2$ & 31.92 \\
\hline Ouvigo & - & Paleo. & 42 & 0 & 7 & 45 & $2881 / 4$ & $2 \frac{1}{2}$ & 14.99 \\
\hline Fraga & Fortunatus & Paleo. & 41 & 33 & 0 & 19 & 42 & 7 & 39.16 \\
\hline Serós/Bolvalar & - & Paleo. & 41 & 27 & 0 & 25 & $871 / 4$ & $23 / 4$ & 3.83 \\
\hline Constantí & $V . R$. Centcelles & Paleo. & 41 & 9 & 1 & 13 & 19 & $1 \frac{1}{4}$ & 46.21 \\
\hline Tarragona & Sta. María & Paleo. & 41 & 6 & -1 & 15 & $56^{1 / 4}$ & 0 & 24.36 \\
\hline Tarragona & S. Fructuoso & Paleo. & 41 & 6 & -1 & 14 & 76 & 2 & 11.63 \\
\hline Carranque & - & Paleo. & 40 & 11 & 3 & 57 & 211 & $-01 / 2$ & -41.9 \\
\hline Es Cap des Port & - & Paleo. & 40 & 1 & 4 & 7 & $72 \frac{1}{2}$ & 6 & 17.14 \\
\hline Son Bou & - & Paleo. & 39 & 53 & -4 & 17 & $1251 / 2$ & 11 & -18.36 \\
\hline Illa del rei & - & Paleo. & 39 & 53 & -4 & 17 & 102 & 1 & -8.79 \\
\hline Es Fornás de Torelló & - & Paleo. & 39 & 52 & -4 & 13 & 100 & $-01 / 4$ & -8.27 \\
\hline Son Peretó & - & Paleo. & 39 & 35 & -3 & 15 & 112 & 0 & -17.08 \\
\hline Monforte & V. R. Torre de Palma & Paleo. & 39 & 3 & 7 & 29 & $74^{1} / 2$ & $1 \frac{1}{2}$ & 12.71 \\
\hline Mérida & Casa Herrera & Paleo. & 38 & 57 & 6 & 17 & $74 \frac{1}{2}$ & $01 / 2$ & 12.00 \\
\hline Mérida & S.Eulalia(Martirium) & Paleo. & 38 & 55 & 6 & 19 & 86 & 0 & 2.75 \\
\hline Mérida & S.Eulalia(B) & Paleo. & 38 & 55 & 6 & 19 & $88^{1 / 2}$ & 0 & 0.81 \\
\hline Mérida & Xenodoquio & Paleo. & 38 & 55 & 6 & 19 & $881 / 2$ & 0 & 0.81 \\
\hline Sao Cucufate & - & Paleo. & 38 & 13 & 7 & 50 & $1281 / 2$ & 1 & -29.04 \\
\hline Cástulo & Martirium & Paleo. & 38 & 2 & 3 & 37 & 192 & $01 / 2$ & -50.79 \\
\hline Algezares & - & Paleo. & 37 & 56 & 1 & 6 & $146^{1 / 2}$ & 19 & -24.98 \\
\hline Tejada & V.R. Bruñel & Paleo. & 37 & 53 & 3 & 5 & 100 & $71 / 2$ & -3.45 \\
\hline Mosteiro & S. Salvador & Paleo. & 37 & 47 & 7 & 43 & $92 \frac{1}{2}$ & 3 & -0.29 \\
\hline Mértola & Baptisterio 1 & Paleo. & 37 & 38 & 7 & 39 & $81 \frac{1}{4}$ & 2 & 8.05 \\
\hline Mértola & Baptisterio 2 & Paleo. & 37 & 38 & 7 & 39 & $931 / 4$ & 2 & -1.45 \\
\hline Mértola & Basílica & Paleo. & 37 & 38 & 7 & 39 & $102^{1 / 4}$ & $31 / 2$ & -7.67 \\
\hline Gerena & - & Paleo. & 37 & 31 & 6 & 8 & $75^{3} / 4$ & 0 & 10.82 \\
\hline Mijangos & Sta. María & Visig. & 42 & 49 & 3 & 25 & $951 / 4$ & 1 & -3.41 \\
\hline Valdelateja & Sta. Centola y Elena & Visig. & 42 & 46 & 3 & 45 & $891 / 2$ & $4 \frac{1}{2}$ & 3.30 \\
\hline Espierre & Sta. María & Visig. & 42 & 37 & 0 & 17 & $92^{3} / 4$ & 9 & 4.01 \\
\hline San Pelay & Cripta & Visig. & 42 & 37 & 0 & 17 & $631 / 4$ & $51 / 2$ & 23.13 \\
\hline Tricio & $\begin{array}{l}\text { Sta. María de los } \\
\text { Arcos }\end{array}$ & Visig. & 42 & 24 & 2 & 42 & $901 / 2$ & $13 / 4$ & 0.59 \\
\hline VentasBlancas & Sta. María & Visig. & 42 & 21 & 2 & 17 & 77 & $2 \frac{1}{2}$ & 11.09 \\
\hline Suso & - & Visig. & 42 & 20 & 2 & 52 & $152^{1 / 2}$ & 6 & -35.70 \\
\hline Suso & capilla & Visig. & 42 & 20 & 2 & 52 & $62 \frac{1}{2}$ & 17 & 31.52 \\
\hline S.Vicente & S.Vicente & Visig. & 42 & 20 & 3 & 9 & $621 / 2$ & 17 & 31.52 \\
\hline
\end{tabular}


Table 1 continued

\begin{tabular}{|c|c|c|c|c|c|c|c|c|c|}
\hline Place & Monument & Period & $\begin{array}{l}\varphi \\
(\%)\end{array}$ & & $\begin{array}{l}\lambda \\
\left({ }^{\circ} /{ }^{\prime}\right)\end{array}$ & & a $\left({ }^{\circ}\right)$ & $\mathrm{h}\left({ }^{\circ}\right)$ & $\delta\left({ }^{\circ}\right)$ \\
\hline Panxón & S. Xoan & Visig & 42 & 8 & 8 & 49 & $95^{1 / 2}$ & $31 / 2$ & -1.86 \\
\hline Quintanilla de las Viñas & Sta. María & Visig. & 42 & 7 & 3 & 30 & $103 \frac{1}{2}$ & $0^{1 / 2}$ & -9.96 \\
\hline Ambía & Sta.Eufemia & Visig. & 42 & 3 & 7 & 42 & 89.8 & 5 & 3.38 \\
\hline Palencia & Cripta & Visig. & 42 & 0 & 4 & 43 & $581 / 4$ & 0 & 22.56 \\
\hline Boada & S. Juliá & Visig. & 41 & 59 & 3 & 7 & $873 / 4$ & 0 & 1.32 \\
\hline Silos & Sta. Cecilia & Visig. & 41 & 58 & 3 & 29 & 83 & $51 / 2$ & 8.77 \\
\hline Baños & S. Juan & Visig. & 41 & 55 & 4 & 28 & $893 / 4$ & $01 / 2$ & 0.20 \\
\hline Bande & Sta. Comba & Visig. & 41 & 43 & 8 & 4 & $911 / 4$ & 2 & 0.23 \\
\hline Manresa & $\begin{array}{l}\text { Sta. María des } \\
\text { Matadars }\end{array}$ & Visig. & 41 & 41 & -1 & 52 & $913 / 4$ & 8 & 3.94 \\
\hline Egara & Sta. Maria & Visig. & 41 & 33 & -2 & 0 & 83 & 0 & 4.85 \\
\hline Egara & S. Miguel & Visig. & 41 & 33 & -2 & 0 & 85 & 0 & 3.36 \\
\hline Egara & St. Pere & Visig. & 41 & 33 & -2 & 0 & $84 \frac{1}{2}$ & 0 & 3.73 \\
\hline Braga & S. Fructuoso & Visig. & 41 & 33 & 8 & 26 & $671 / 2$ & 6 & 20.70 \\
\hline Abrera & Sant Hilari & Visig. & 41 & 31 & -1 & 54 & 59 & $4 \frac{1}{2}$ & 25.75 \\
\hline Sant Cugat del Vallés & Sant Cugat & Visig. & 41 & 28 & -2 & 5 & 63 & $0^{1 / 2}$ & 19.90 \\
\hline Pedro & Virgen del Val & Visig. & 41 & 19 & 3 & 11 & $89 \frac{1}{2}$ & $51 / 2$ & 3.90 \\
\hline Recópolis & - & Visig. & 40 & 19 & 2 & 53 & 107 & $13 / 4$ & -11.93 \\
\hline Idanha-a-Velha & Sei & Visig. & 39 & 59 & 7 & 8 & $951 / 2$ & $1 \frac{1}{2}$ & -3.47 \\
\hline Segóbriga & Basílica & Visig. & 39 & 53 & 2 & 48 & $104 \frac{1}{2}$ & 3 & -9.36 \\
\hline Toledo & S. Salvador & Visig. & 39 & 51 & 4 & 1 & $132 \frac{3}{4}$ & 0 & -31.84 \\
\hline Valeria & Sey & Visig. & 39 & 48 & 2 & 9 & $104^{3} / 4$ & 0 & -11.65 \\
\hline Melque & Sta. María & Visig & 39 & 45 & 4 & 22 & $65^{1 / 2}$ & 0 & 18.20 \\
\hline Casalgordo & S. Pedro & Visig. & 39 & 36 & 3 & 59 & 67 & $-0^{1 / 2}$ & 16.72 \\
\hline Nazaré & Sao Giao & Visig. & 39 & 33 & 9 & 5 & 131 & $10^{1 / 2}$ & -22.47 \\
\hline Valencia & Catedral & Visig. & 39 & 28 & 0 & 22 & $96^{3} / 4$ & 0 & -5.57 \\
\hline Valencia & Martirum & Visig. & 39 & 28 & 0 & 22 & $102 \frac{1}{4}$ & 0 & -9.80 \\
\hline Cáceres & Santa Olalla & Visig. & 39 & 24 & 6 & 23 & 123 & $2 \frac{1}{2}$ & -23.31 \\
\hline Trampal & Sta. Lucía & Visig. & 39 & 9 & 6 & 13 & $861 / 2$ & -1 & 1.55 \\
\hline Oretum & Sta. Ana & Visig. & 38 & 48 & 3 & 30 & $801 / 4$ & 0 & 8.14 \\
\hline Oretum & S. Bartolome & Visig. & 38 & 48 & 3 & 30 & $82 \frac{1}{2}$ & 0 & 6.20 \\
\hline Valdecebadar & - & Visig. & 38 & 44 & 7 & 5 & $891 / 4$ & 1 & 1.07 \\
\hline Tolmo & - & Visig. & 38 & 28 & 1 & 42 & 85 & $1 \frac{1}{2}$ & 4.63 \\
\hline Elche & Basílica & Visig. & 38 & 14 & 0 & 41 & $931 / 4$ & 0 & -2.84 \\
\hline El Germo & - & Visig. & 38 & 12 & 4 & 57 & 101 & $1 \frac{1}{4}$ & -8.08 \\
\hline Córdoba & S. Julián & Visig. & 37 & 53 & 4 & 46 & $92 \frac{1}{2}$ & 0 & -2.33 \\
\hline Basti & - & Visig. & 37 & 30 & 2 & 44 & $931 / 2$ & $1 \frac{1}{2}$ & -2.13 \\
\hline $\begin{array}{l}\text { Montinho dos } \\
\text { Laranjeiros }\end{array}$ & - & Visig. & 37 & 24 & 7 & 27 & $1291 / 4$ & $51 / 2$ & -26.30 \\
\hline S.Pedro de Alcántara & - & Visig. & 36 & 28 & 4 & 59 & $911 / 4$ & $-01 / 4$ & -1.42 \\
\hline Santianés & S. Juan & Astur. & 43 & 30 & 6 & 5 & $71^{1 / 4}$ & 3 & 15.12 \\
\hline Bedriana & S. Andrés & Astur. & 43 & 30 & 5 & 26 & $91 \frac{1}{2}$ & $1 \frac{1}{4}$ & -0.49 \\
\hline
\end{tabular}


Table 1 continued

\begin{tabular}{|c|c|c|c|c|c|c|c|c|c|}
\hline Place & Monument & Period & $\begin{array}{l}\varphi \\
(\%)\end{array}$ & & $\begin{array}{l}\lambda \\
\left({ }^{\circ} /\right)\end{array}$ & & a $\left({ }^{\circ}\right)$ & $\mathrm{h}\left({ }^{\circ}\right)$ & $\delta\left({ }^{\circ}\right)$ \\
\hline Priesca & S. Salvador & Astur. & 43 & 29 & 5 & 21 & 83 & $51 / 4$ & 8.19 \\
\hline Gobiendes & Santiago & Astur. & 43 & 28 & 5 & 14 & $783 / 4$ & -1 & 7.00 \\
\hline Valdedios & S. Salvador & Astur. & 43 & 26 & 5 & 30 & $571 / 4$ & 20 & 36.67 \\
\hline Arbazal & Sta. María & Astur. & 43 & 25 & 5 & 29 & 82 & $71 / 2$ & 10.87 \\
\hline Nora & S. Pedro & Astur. & 43 & 22 & 5 & 57 & $70^{3} / 4$ & $31 / 2$ & 15.75 \\
\hline Oviedo & S. Julián & Astur. & 43 & 22 & 5 & 50 & $73 \frac{3}{4}$ & $-01 / 2$ & 11.00 \\
\hline Oviedo & S. María del Naranco & Astur. & 43 & 22 & 5 & 51 & $92 \frac{1}{2}$ & $61 / 4$ & 2.03 \\
\hline Oviedo & S.Miguel de Lillo & Astur. & 43 & 22 & 5 & 52 & $651 / 2$ & $15^{1 / 2}$ & 27.77 \\
\hline Oviedo & Sto. Tirso & Astur. & 43 & 21 & 5 & 50 & $74 \frac{3}{4}$ & 0 & 10.53 \\
\hline Oviedo & Cámara Santa & Astur. & 43 & 21 & 5 & 50 & $72 \frac{1}{2}$ & 0 & 12.22 \\
\hline Bendones & Sta. María & Astur. & 43 & 20 & 5 & 48 & $88^{1} / 2$ & 4 & 3.35 \\
\hline Tuñón & Sto. Adriano & Astur. & 43 & 17 & 5 & 58 & $591 / 2$ & $15^{1 / 2}$ & 32.16 \\
\hline Lena & Sta. Cristina & Astur. & 43 & 7 & 5 & 48 & $433 / 4$ & 11 & 39.77 \\
\hline Francelos & S. Ginés & Astur. & 42 & 16 & 8 & 9 & $701 / 2$ & 2 & 15.47 \\
\hline Cuevas & Ntra. Señora Cerro & Astur. & 42 & 16 & 3 & 32 & $106^{1 / 2}$ & 3 & -10.22 \\
\hline Bostronizo & S. Román de Moroso & Moza. & 43 & 13 & 4 & 3 & 77 & 9 & 15.54 \\
\hline Lebeña & Sta. María & Moza. & 43 & 12 & 4 & 35 & $871 / 2$ & $181 / 2$ & 14.29 \\
\hline Retorta & Sta. Cruz & Moza. & 43 & 1 & 7 & 41 & $873 / 4$ & 2 & 2.80 \\
\hline Berbetouros & S. Miguel & Moza. & 42 & 55 & 7 & 48 & $831 / 2$ & $0^{1 / 2}$ & 4.77 \\
\hline Santiago & Catedral & Moza. & 42 & 52 & 8 & 55 & 86 & $01 / 2$ & 2.94 \\
\hline Santiago & Corticela & Moza. & 42 & 50 & 8 & 32 & 79 & $1 \frac{1 / 4}{2}$ & 8.64 \\
\hline Olleros & S. Pelayo & Moza. & 42 & 45 & 4 & 16 & 96 & 2 & -3.25 \\
\hline Olleros & Stos. Justo y Pastor & Moza. & 42 & 45 & 4 & 16 & 62 & 0 & 19.75 \\
\hline Villatuerta & S. Miguel & Moza. & 42 & 39 & 2 & 0 & $78^{1 / 2}$ & 1 & 9.11 \\
\hline León & S. Salvador de Palat & Moza. & 42 & 35 & 5 & 34 & $671 / 2$ & 0 & 15.95 \\
\hline León & S. Isidoro & Moza. & 42 & 35 & 5 & 34 & $661 / 4$ & 0 & 16.94 \\
\hline Escalada & S. Miguel & Moza. & 42 & 34 & 5 & 18 & $981 / 2$ & 0 & -6.64 \\
\hline S.Tomás de las Ollas & S.Tomás & Moza. & 42 & 33 & 6 & 34 & $891 / 4$ & 4 & 3.06 \\
\hline S. Juan de la Peña & S. Juan & Moza. & 42 & 30 & 0 & 40 & $122 \frac{1}{2}$ & 0 & -23.76 \\
\hline Andorra & Sta.Coloma & Moza. & 42 & 29 & -1 & 29 & $641 / 4$ & $61 / 2$ & 23.16 \\
\hline Peñalba de Santiago & Santiago & Moza. & 42 & 25 & 6 & 32 & 82 & 22 & 20.34 \\
\hline Sta. Coloma & Sta. Coloma & Moza. & 42 & 22 & 2 & 39 & $751 / 2$ & $51 / 2$ & 14.30 \\
\hline Viñao & S. Pedro & Moza. & 42 & 22 & 7 & 59 & $901 / 2$ & 9 & 5.53 \\
\hline Fontefría & S. Xiao & Moza. & 42 & 22 & 7 & 56 & $733 / 4$ & 1 & 12.34 \\
\hline Suso & - & Moza. & 42 & 20 & 2 & 52 & $56^{1 / 2}$ & 17 & 35.92 \\
\hline Sta. & Sta. Ouxea & Moza. & 42 & 20 & 7 & 55 & 110 & 10 & -7.65 \\
\hline Torrecilla de Cameros & S. Andrés & Moza. & 42 & 15 & 2 & 37 & $84 \frac{1}{2}$ & $81 / 2$ & 9.69 \\
\hline Torrecilla de Cameros & S. Pedro & Moza. & 42 & 15 & 2 & 39 & 96 & 15 & 5.66 \\
\hline PalolSabaldoria & Sto. Tomás & Moza. & 42 & 14 & -2 & 56 & 65 & $1 \frac{1}{2}$ & 19.00 \\
\hline Arnedillo & Sta. Maria de Peñalba & Moza. & 42 & 12 & 2 & 13 & $791 / 2$ & $-01 / 4$ & 7.16 \\
\hline Pazoo & S. Martín & Moza. & 42 & 10 & 7 & 49 & $1001 / 4$ & 3 & -5.70 \\
\hline Celanova & S. Miguel & Moza. & 42 & 9 & 7 & 57 & $903 / 4$ & 2 & 0.58 \\
\hline
\end{tabular}


Table 1 continued

\begin{tabular}{|c|c|c|c|c|c|c|c|c|c|}
\hline Place & Monument & Period & $\begin{array}{l}\varphi \\
(\%)\end{array}$ & & $\begin{array}{l}\lambda \\
(\%)\end{array}$ & & a $\left({ }^{\circ}\right)$ & $\mathrm{h}\left({ }^{\circ}\right)$ & $\delta\left({ }^{\circ}\right)$ \\
\hline Ampurias & Sta. Margarita & Moza. & 42 & 7 & -3 & 6 & 69 & $4^{1 / 2}$ & 18.40 \\
\hline Berga & S. Quirze & Moza. & 42 & 6 & -1 & 52 & $91^{3 / 4}$ & 8 & 3.99 \\
\hline Retortillo & Sta. María & Moza. & 42 & 4 & 4 & 1 & $82^{1} / 2$ & $01 / 2$ & 5.57 \\
\hline Berga & S. Vicent & Moza. & 42 & 3 & -1 & 52 & $79^{1 / 4}$ & 2 & 9.11 \\
\hline Xinzo & S. Mariña & Moza. & 42 & 3 & 7 & 43 & $112^{1 / 4}$ & $03 / 4$ & -16.11 \\
\hline Obiols & S. Vicenç & Moza. & 42 & 3 & -1 & 51 & $80^{1 / 2}$ & 2.3 & 8.40 \\
\hline Hortigüela & S. Pedro el Viejo & Moza. & 42 & 2 & 3 & 28 & 106 & $3 \frac{1}{2}$ & -9.55 \\
\hline Palencia & Cripta & Moza. & 42 & 0 & 4 & 32 & 61 & 0 & 20.77 \\
\hline Quintanar & S. Andrés & Moza. & 41 & 59 & 3 & 1 & $961 Q$ & 9 & 1.53 \\
\hline Mosteiro & - & Moza. & 41 & 59 & 7 & 47 & $88^{1 / 4}$ & $-0^{1 / 2}$ & 0.50 \\
\hline Boada & S. Juliá & Moza. & 41 & 59 & 3 & 7 & $75^{3} / 4$ & 0 & 10.15 \\
\hline Mixós & S. María & Moza. & 41 & 57 & 7 & 27 & $651 / 4$ & $31 / 2$ & 20.34 \\
\hline Barriosuso & S. Cecilia & Moza. & 41 & 57 & 3 & 28 & $841 / 2$ & $2^{1 / 2}$ & 5.58 \\
\hline Antigüedad & Villella & Moza. & 41 & 55 & 4 & 7 & 73 & $31 / 2$ & 14.80 \\
\hline Trigueros del Valle & $\begin{array}{l}\text { Nta. Señora del } \\
\text { Castillo }\end{array}$ & Moza. & 41 & 49 & 4 & 39 & $63^{1 / 2}$ & $-01 / 2$ & 18.57 \\
\hline Tábara & S. Salvador & Moza. & 41 & 49 & 5 & 57 & $861 / 4$ & $-01 / 2$ & 1.99 \\
\hline Tabérnolas & S. Feliu de Savassona & Moza. & 41 & 47 & -2 & 20 & $671 / 2$ & $1 \frac{1}{2}$ & 17.33 \\
\hline Coruña del Conde & Ermita & Moza. & 41 & 45 & 3 & 23 & $751 / 4$ & $01 / 2$ & 11.00 \\
\hline Manresa & Sta. MaríaMatadars & Moza. & 41 & 41 & -1 & 52 & 85 & $14 \frac{1}{2}$ & 13.23 \\
\hline Wamba & Sta. María & Moza. & 41 & 40 & 4 & 55 & $671 / 4$ & 1 & 17.20 \\
\hline Mazote & S. Cebrián & Moza. & 41 & 40 & 5 & 8 & $95 \frac{3}{4}$ & 4 & -1.75 \\
\hline S. Salvador Guardiola & St. Père de Brunet & Moza. & 41 & 40 & -1 & 47 & 98 & 2 & -4.83 \\
\hline Esparraguera & Sta. Margarida Cairat & Moza. & 41 & 34 & 1 & 51 & 69 & $61 / 4$ & 19.71 \\
\hline Cabrils & St. Cristófor & Moza. & 41 & 30 & -2 & 23 & 74 & $-01 / 4$ & 11.31 \\
\hline Gormaz & S. Miguel & Moza. & 41 & 29 & 3 & 0 & 81 & -1 & 5.51 \\
\hline Berlanga & S. Baudelio & Moza. & 41 & 25 & 2 & 47 & 54 & 1 & 26.59 \\
\hline S. Miquel de Olerdola & S. Miquel & Moza. & 41 & 19 & -1 & 43 & 105 & $4 \frac{1}{2}$ & -8.28 \\
\hline Coimbra & Sao Pedro de Lorousa & Moza. & 40 & 19 & 7 & 55 & $82 \frac{1}{2}$ & $31 / 4$ & 7.67 \\
\hline Ardales & Bobastro & Moza. & 36 & 54 & 4 & 46 & 75 & 20 & 23.54 \\
\hline Gavín & S. Bartolome & Serrab. & 42 & 37 & 0 & 17 & $92^{1 / 4}$ & $22 \frac{1}{2}$ & 13.41 \\
\hline Basarán & S. Urbez & Serrab. & 42 & 37 & 0 & 17 & 94 & 0 & -3.33 \\
\hline S.Pelay & Iglesia & Serrab. & 42 & 37 & 0 & 17 & $681 / 4$ & $51 / 2$ & 19.55 \\
\hline Orós & S. Xuan & Serrab. & 42 & 35 & 0 & 18 & $981 / 4$ & 16 & 4.83 \\
\hline Oliván & S. Martín & Serrab. & 42 & 34 & 0 & 18 & $123^{3 / 4}$ & $81 / 2$ & -17.82 \\
\hline Susín & St. Eulalia & Serrab. & 42 & 34 & 0 & 18 & $104 \frac{1}{4}$ & $41 / 2$ & -7.46 \\
\hline Larrede & S. Pedro & Serrab. & 42 & 33 & 0 & 19 & $973 / 4$ & 16 & 5.18 \\
\hline Larrede & S. Juan & Serrab. & 42 & 33 & 0 & 19 & $961 / 4$ & 23 & 10.95 \\
\hline Satue & S. Andrés & Serrab. & 42 & 31 & 0 & 19 & $116^{1 / 4}$ & 20 & -4.35 \\
\hline Isún & Asunción & Serrab. & 42 & 30 & 0 & 19 & $105^{1 / 4}$ & 6 & -7.12 \\
\hline Arto & S. Martín & Serrab. & 42 & 27 & 0 & 25 & $791 / 4$ & 3 & 9.79 \\
\hline Lasieso & S. Pedro & Serrab. & 42 & 25 & 0 & 26 & $96^{1 / 2}$ & $1^{1 / 2}$ & -4.01 \\
\hline
\end{tabular}


Table 1 continued

\begin{tabular}{lllllllllr}
\hline Place & Monument & Period & $\begin{array}{l}\varphi \\
\left({ }^{\circ} /\right)\end{array}$ & $\begin{array}{l}\lambda \\
\left({ }^{\circ} /\right)\end{array}$ & a $\left({ }^{\circ}\right)$ & $\mathrm{h}\left({ }^{\circ}\right)$ & $\delta\left({ }^{\circ}\right)$ \\
\hline Ordovés & S. Martín & Serrab. & 42 & 24 & 0 & 21 & $833 / 4$ & 5 & 7.87 \\
Yeste & - & Serrab. & 42 & 23 & 0 & 41 & 94 & 3 & -1.09 \\
Rasal & S. Juan & Serrab. & 42 & 22 & 0 & 36 & 80 & 7 & 12.00 \\
\hline
\end{tabular}

The table shows for each monument the location, the identification of the structure, the latitude and longitude ( $\varphi$ and $\lambda$ ), its azimuth (a) from outside looking in, and the angular height of the horizon (h) in that direction, and the corresponding declination $(\delta)$. Measurements obtained from satellite images and/or Digital Terrain Models are indicated in italics

change in the orientation, such as St. María des Matadars (in Catalonia), or a new church was often built on the site of a previous one, destroyed during the Muslim invasion or for other causes, such as the Cathedral of Santiago de Compostela. In those few cases, each of the orientations of the church was measured and may appear several times in Table 1 for each entry.

Table 1 presents our comprehensive data sample. It comprises a total of 167 churches in total measured in a series of campaigns starting from 2008 in Asturias until 2014. Our measurements include thirty-two Palaeo-Christian churches (Fig. 3) defined as those built before the establishment of the Germanic kingdoms in the Peninsula by the fifth century A.D. There are forty-seven Visigoth churches, including under this definition also those churches possibly built during the Byzantine domination of the south of the Peninsula. We have also measured seventeen buildings that were in some way related to the Kingdom of Asturias, plus seventy-one commonly referred to as Mozarabic in style and covering a clear time period. These include fifteen churches for the Serrablo valley in northern Aragón, which are identified in Table 1. It must be stressed here that some of the churches have allegedly been included in different periods by different authors. The division between Visigoth and Mozarabic churches is particularly worrisome because the styles are sometimes so close that they are difficult to distinguish unless the original date of construction is clear. Hence, it has been used with caution here. We refer the interested reader to Utrero Agudo (2006) for the division used here, which is mostly based on architectonic and epigraphic data (see also Fontaine 1978a, b; Palol i Salellas 1991).

The orientation data presented in Table 1 were collected mostly in the field (see Fig. 7) using high precision compasses and clinometers, and correcting for magnetic declination. Magnetic alterations are not expected in most of the Iberian Peninsula. The field measurements included in Table 1 have an average error of $1 / 2^{\circ}$ in azimuth and $12^{\circ}$ in horizon altitude, which translates into an error of $\sim 34^{\circ}$ in declination. A number of measurements were obtained by satellite images or from ortho photos. These are indicated in Table 1 in italic characters. In those particular cases, the angular height of the horizon has been calculated either from the «heywhatsthat.com $\gg$ site or using the Google Earth capabilities. The different errors introduced in these procedures increase to about $1 \frac{1}{2^{\circ}}$ in declination. These errors 


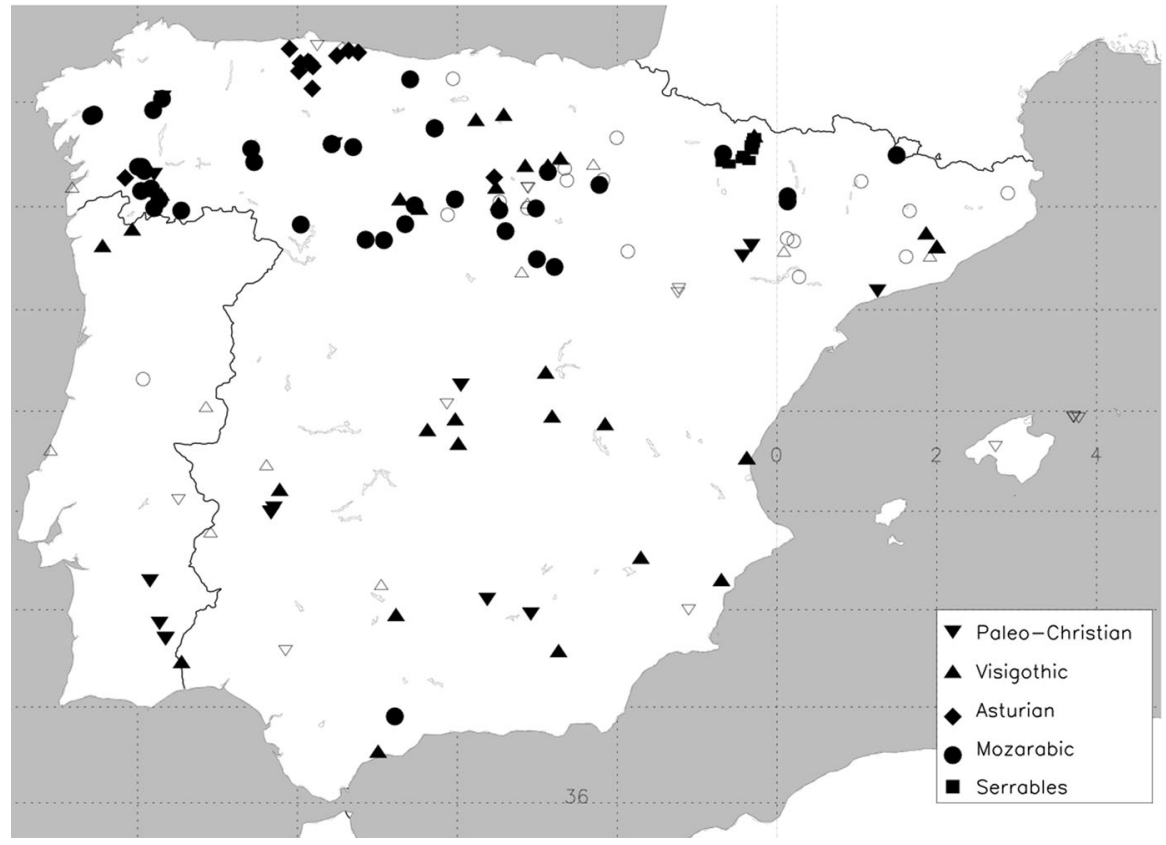

Fig. 7 Map of the sites mentioned presented in Table 1 and discussed within the text where the data have been collected. The different symbols indicate the different pre-Romanesque periods in the Iberian Peninsula (see inset). Open symbols are for data obtained through satellite or orthophotographic measurements

are treated separately when building the histograms, considering a bin-width of twice the error for each of these particular entries.

As in previous works (see, for example, González-García and Belmonte 2014b), we have used an appropriate smoothing of the declination by a function called 'kernel' to produce the kernel density distribution (hereafter KDD) to build the histograms. For each declination entry, we multiply the value of the declination by the kernel function with a given passband or width. For this process, an Epanechnikov kernel is employed with a bandwidth of twice our estimated error in declination. Finally, all the kernels for each declination entry are summed up to form the KDD for our data. To be able to say whether a measurement is significant, we use a normalized relative frequency to scale our KDDs or histograms. To do so, we divide the number of occurrences of a given value by the mean number of occurrences for that sample; this is equivalent to dividing or comparing with the results of a uniform distribution of the same size as our data sample, and with a value equal to the mean of our data.

\section{Global Statistics}

Figure 8 shows the orientation diagram for all 167 orientations considered in our sample. Each orientation is indicated by a short solid line inside the circle that 
a
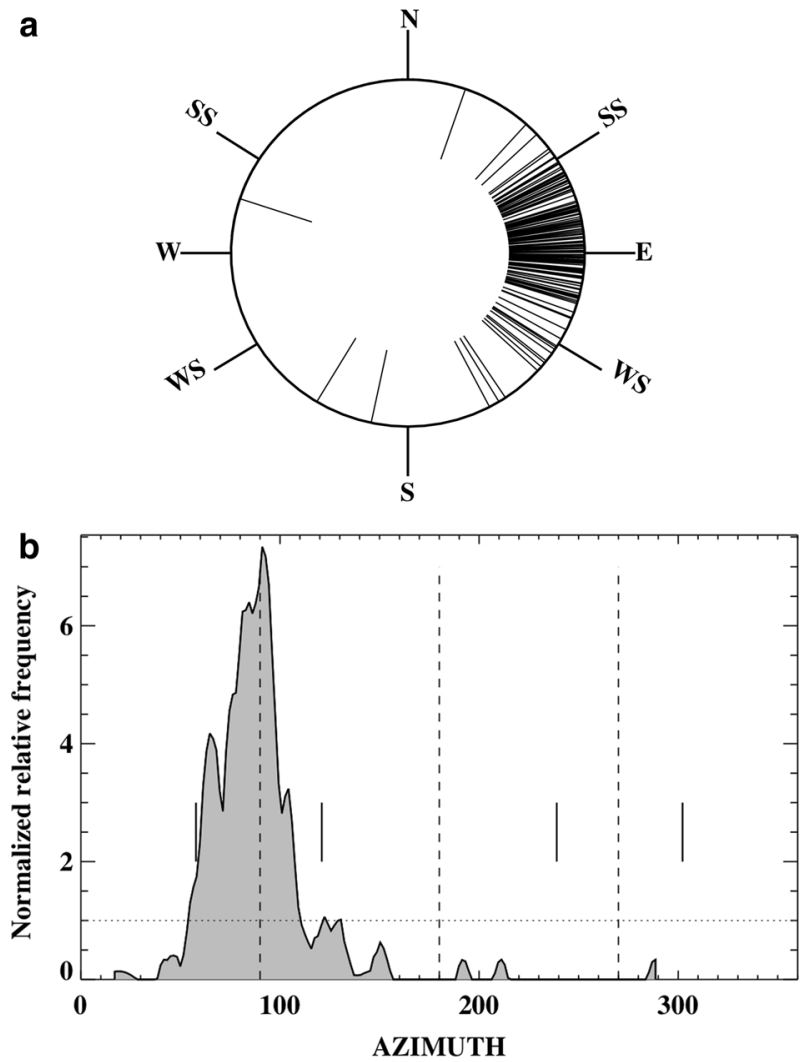

Fig. 8 Left Orientation diagram for all churches in our sample. Out of 167 measurements, only 18 are beyond the solar range at sunrise, indicated by the SS and WS marks, standing for Summer Solstice and Winter Solstice at $41^{\circ} \mathrm{N}$-average latitude of the data-respectively. Right Histogram of azimuth for the 167 measurements. The largest concentration of orientations is towards the east. There is a clear secondary maximum at orientations close to $60^{\circ}$. See text for further details

encompasses the different direction on the horizon, with the short solid lines outside the circle indicating particular directions, such as the cardinal directions and the solar limits (i.e., the extreme positions of sunrise and sunset at the solstices; WS stands for winter solstice and SS for summer solstice). Figure 8 shows that most of our orientations are directed towards the eastern half of the horizon. Just three orientations happen on the western half and all of them are from Palaeo-Christian times (Ouvigo, Carranque and Cástulo) and only fourteen are outside the limits of the solar rising range (nine Palaeo-Christian, two Visigothic, one Asturian and one Mozarabic). Most of the churches in our sample tend to be orientated within the solar rising range, with a tendency to be orientated north of due east (ninety-eight churches are orientated in this area, and this accounts for $60 \%$ of our sample). It should be noted that in this first analysis we do not take into account the horizon altitude; thus, some of these churches will indeed be orientated within the solar range when considering the declination. 
Figure 8 also shows an azimuthal histogram for all the churches in our sample. Thus, the conclusions from the above analysis can be refined. This histogram shows three, perhaps four, maxima of above $99 \%$ significance, indicated by a value of 3 in this diagram. The principal maxima are near an azimuth of $90^{\circ}$ and show a possible double nature; however the fact that we do not consider the horizon height in this plot hampers our conclusions. A secondary maximum appears at azimuth $67^{\circ}$, clearly different from any relation with the summer solstice sunrise. Finally, the third peak appears at a value of $105^{\circ}$.

Figure $1 \mathrm{~b}$ shows the declination histogram, where we do include the effect of the horizon height and thus we can compare directly with the solar hypothesis. Such is indicated by the two vertical solid lines, which provide the limits of the sun at the solstices. The horizontal dotted line indicates a measure of the statistical confidence of the maxima; all peaks above this line can be considered significant beyond the $99 \%$ confidence level. The main maximum has a peak at $3.37^{\circ}$, and a significant spread. If we translate such declination value into solar rising dates this would be 29 March or 14 September in the Gregorian proleptic calendar. There is a second maximum at a declination $10.03^{\circ}$ (corresponding to 16 April or 27 August). Finally there are two other maxima at values of $18.51^{\circ}$ and $-10.57^{\circ}$, corresponding to 14 May or 30 July, and 21 February or 21 October, again in the Gregorian proleptic calendar.

It is interesting to note that, although a large number of orientations seem consistent with the solar rising hypothesis, there seems to be an avoidance of orientations compatible with the solstices. This is at odds with what should be expected if we take the solar rising hypothesis to its extreme, assuming that any date during the year could be used to orientate the church. Based on this, there seems to be a preference towards dates between the spring equinox and autumn equinox in what we may call the summer months.

\section{Group by Group Statistics}

In order to see the possible differences between the various epochs, and as an exercise to try to disentangle the possible meaning of some of the maxima above highlighted, we have considered the data separated according to the chronological divisions presented in "The Church in Iberia in pre-Romanesque times". We thus perform in the following a detailed analysis in three groups: (a) Palaeo-Christian plus Visigothic churches, (b) Asturian churches and (c) Mozarabic plus Serrables churches.

\section{Palaeo-Christian and Visigothic Churches}

Figure 9a shows the orientation diagram of the thirty-two Palaeo-Christian churches considered in our sample. As previously mentioned, it is clear that, although there is a preference for orientations towards the eastern half of the horizon, it is not rare also to find orientations to the west and especially a high incidence of orientations outside the sunrise range. This is the only period where we find such an outcome. In this case, these could be understood in the context of the formation period of the 
(a)

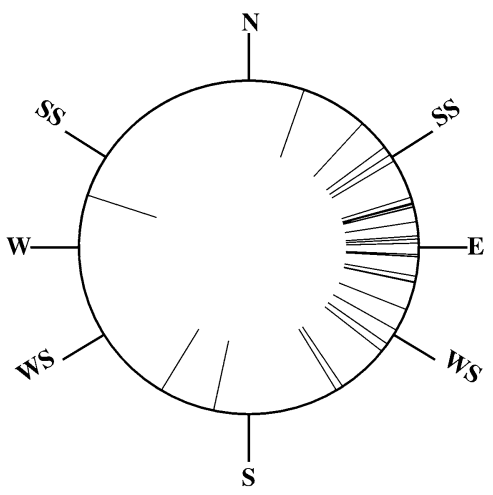

(c)

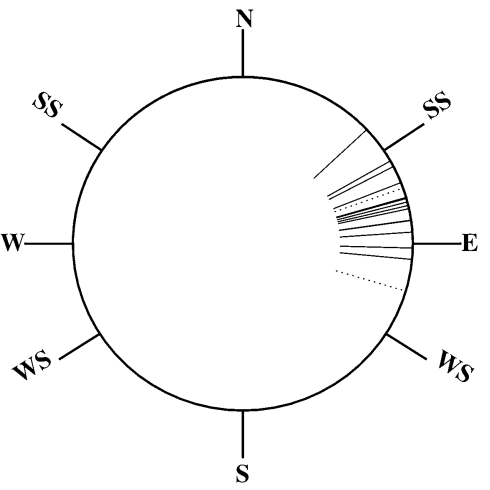

(b)

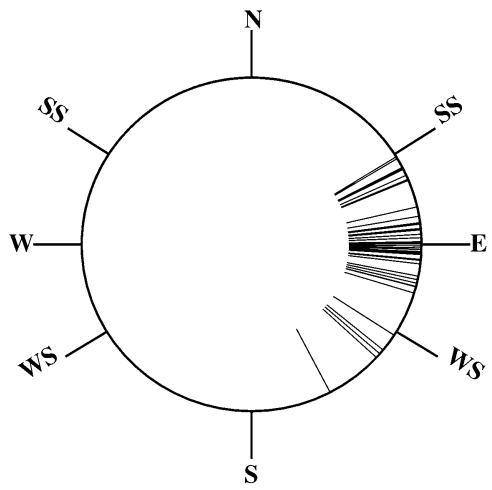

(d)

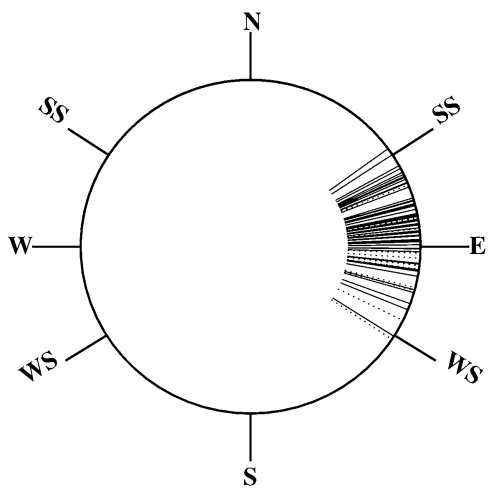

Fig. 9 Orientation diagrams for the groups of monuments in our sample divided chronologically: a thirty-two Palaeo-Christian, b forty-seven Visigoth, c seventeen Asturian, and d fifty-six Mozarabic and fifteen Serrables churches. Palaeo-Christian churches show a preference for orientations within the solar range, although there are a large number of them also outside it. The tendency to have an orientation within the solar range is greater for other later groups. Visigoth churches present a clear concentration towards east, while Asturian churches tend to have orientations north of due east. The dotted lines in this diagram correspond to the only two churches in the sample claimed to be of Asturian origin, which are located outside limits of the present Principality of Asturias. In one case, S. Xes de Francelos (Pontevedra) the orientation is compatible with the bulk of the Asturian churches. However, in the case of Santa María del Cerro (Burgos) the orientation is away from the mean. Mozarabic churches (d) can be divided into the standard Mozarabic and Serrables styles. These last are indicated as dotted lines in the diagram. Although the ranges are similar there is a tendency for Mozarabic churches to be orientated north of east while Serrables churches present a fair number of orientations towards south of due east, presumably for topographic reasons

new religion and its introduction in a new area. The new churches on many occasions make use of already built spaces, such as particular parts of Roman villae (for instance, the villa of Maternus in Carranque, Fig. 3).

Despite of this, Fig. 10a shows that there was a tendency to orientate these churches within the solar range and with a clear focus towards the east, as the principal maximum here has a declination of $1.06^{\circ}$, corresponding to 23-24 March or 19 September in the Gregorian proleptic calendar, or 22 March for the Julian 
(a)

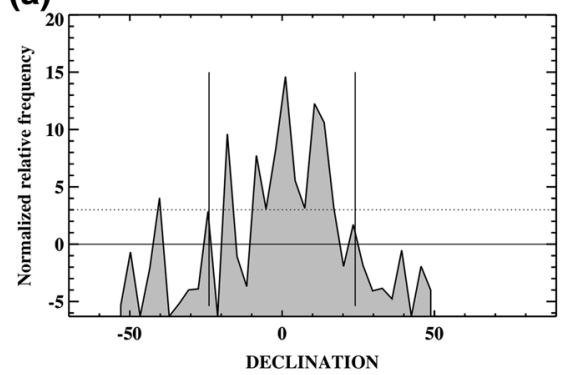

(c)

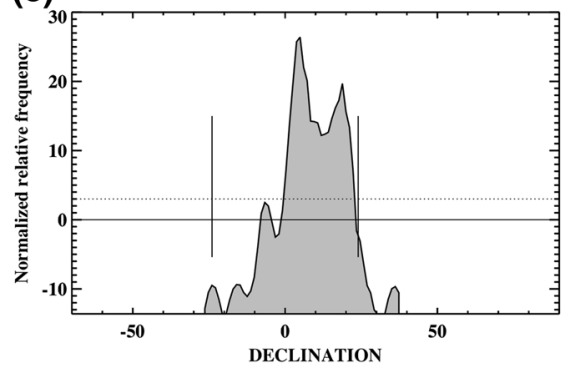

(b)

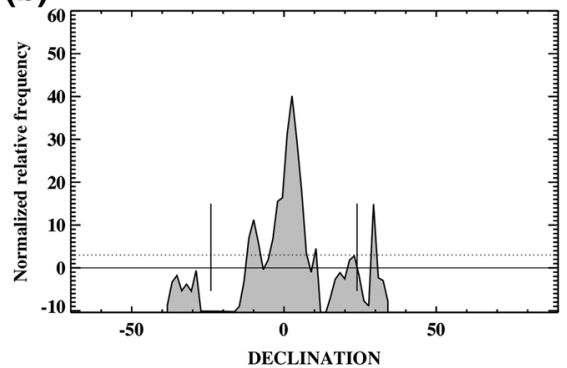

(d)

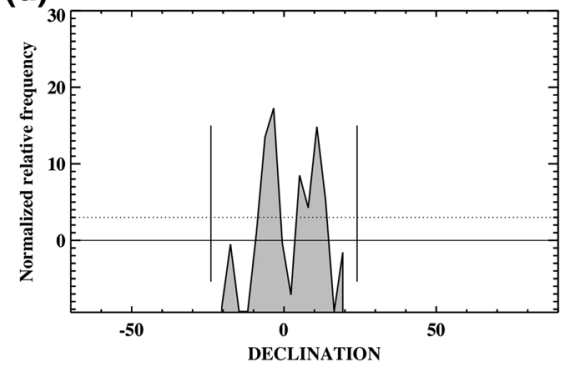

Fig. 10 Declination histograms for Palaeo-Christian (a), Visigoth (b), Mozarabic (c) and Serrables (d) churches. Despite the large spread of orientations for this period, Palaeo-Christian churches present a principal maximum slightly north of due east at $\delta=1.09^{\circ}$. This maximum appears for Visigoth churches at $\delta=2.63^{\circ}$ and for Mozarabic churches at $\delta=4.92^{\circ}$. It is interesting to note that such maxima correspond to sunrise on 25 March in the Julian calendar for the fourth, seventh and tenth centuries, respectively. There are interesting secondary maxima for some of the groups: for example the Visigoth histogram shows one at $\delta=-9.96^{\circ}$, corresponding to 15 October or 21 February with a margin of one day. It is interesting to note that the Cathedra of Saint Peter was celebrated precisely on 22 February. For the Mozarabic churches a secondary maxima appears at $\delta=18.8^{\circ}$, corresponding to 25 July, or 10 May. The first of these dates is the feast of Saint James, whose sepulchre at Compostela was 'discovered' in the ninth century (the pilgrimage route was boosted from this time), becoming a key centre for Western Christianity

calendar in A.D. 400. It should be noted that the sunrise declination for 25 March of that year would be $2.2^{\circ}$. The second maximum appears at $10.5^{\circ}$ of declination, corresponding to 17 April or 26 August proleptic Gregorian (or 18 April or 25 August of the Julian calendar of A.D. 400). A clear-cut comparison of this period can be established with the subsequent Germanic or Visigoth period (Figs. 9b, 10b). There we may see that the orientation in this period is more clearly focused on the sunrise sector of the horizon and in particular towards the east. The principal maximum of the declination histogram appears at $3.07^{\circ}$, such a maximum corresponds to $28 \mathrm{March}$ or 15 September Gregorian proleptic. This is $25 \mathrm{March}$, or 11 September, in A.D. 700 Julian, hence it is clearly equinoctial. The second maximum appears for $-9.82^{\circ}$, or 23 February, 19 October Gregorian proleptic (21 February or 15 October Julian A.D. 700). 


\section{Asturian Churches}

Table 1 includes four churches in addition to those already measured in GonzálezGarcía et al. 2014. Two of the new churches are within the present limits of the region of Asturias and are thus in close geographic relationship to the other thirteen. The orientation of these two churches is quite similar to them. There are claims in the literature (Fontaine 1978a; Valdivieso Ausín 2006) that two other churches could be related, mostly based on style similarity, with those constructed in Asturias in the period between the ends of the ninth to the beginning of the tenth century A.D. One such is the church of San Xes de Francelos in present-day province of Pontevedra. The orientation of this church seems fully compatible with that of those in Asturias.

A different case arises with the second church of alleged Asturian style, the church of Nuestra Señora del Cerro (Burgos). The orientation of this church is quite far from the mean orientation of the Asturian churches by more than twice the standard deviation. This could mean that, according to the orientation, this church is not compatible with the Asturian ones with a $95 \%$ confidence level.

The maximum of the declination distribution for the Asturian churches is at $13.0^{\circ}$, corresponding to 25 April or 18 August in the Gregorian proleptic calendar, and 20 April or 14-15 August Julian A.D. 900; indeed, a peculiar pattern, as already discussed (see also González-García 2014).

\section{Mozarabic and Serrablo Churches}

The orientation diagram for Mozarabic churches is shown in Fig. 9d, including the Valley of Serrablo churches, which are indicated as dotted lines. All the churches but one are within the sunrise arch. It is interesting to note that, if we exclude Serrablo churches from the sample based on the slightly different stylistic and chronological characteristics, the Mozarabic churches show a clear preference for the northern part of the sunrise arch, forty-five churches (80\% of the total) are orientated north of due east. This is not true for the Serrablo churches, where only four churches out of sixteen $(25 \%)$ are north of due east, although, if we consider declination, this percentage rises to $44 \%$ owing to the large horizon height.

Figure 10c shows, on the one hand, the declination histogram for the Mozarabic churches, where the preference for orientations in the summer months is apparent. The main maximum is at $4.04^{\circ}$, corresponding to 30 March and 12 September, Gregorian proleptic (or 25-26 March, 7 September A.D. 1050, Julian). The second maximum is at $18.5^{\circ}$ or 14 May and 30 July Gregorian proleptic, or 9 May, 25 July Julian A.D. 1050. We will come back later to this important datum. On the other hand, the Serrablo churches (Fig. 10d) show two main concentrations, the first at declination $-3.3^{\circ}$ and the second at declination $5^{\circ}$. The first translates into 12 March and 2 October Gregorian proleptic, or 6 March, 26 September Julian AD. 1050. The second corresponds to 2 April and 10 September Gregorian proleptic, or 28 March or 5 September Julian 1050. 


\section{Discussion}

It is interesting to note that our data seems to be in good agreement, in a wider sense, with the results found for other European areas where similar investigations have been carried out. Such results can be read in light of the information provided by the ecclesiastical chronicles during the centuries of the early Middle Ages summarized in the introduction.

The Palaeo-Christian churches display a wide range of orientations in line with the results for other areas throughout the Mediterranean, such as the north of Africa (Belmonte et al. 2007), where there are both orientations towards east and west, that was the case for the early Christian basilicas in the Roman empire, as mentioned by Delgado-Gómez (2006).

However, there seems to be an incipient tendency towards orientating the apse of a significant number of churches in the direction of the rising sun on $25 \mathrm{March}$, the traditional date of the Roman equinox (and the Christian feast of the Annunciation). Such an interest seems to be maintained for all periods, except for the Asturian churches where, although it might be present, it is not the dominant rule. It is particularly interesting to note that such an interest is highlighted by the fact that the orientations appear to point towards this date, when the wandering nature of the Julian calendar is considered, for the main concentration of churches for the Visigoth and Mozarabic churches, as well as the already mentioned PalaeoChristian churches, and arguably the secondary peak of the churches of the Serrablo.

For all cases, it seems clear that there was an avoidance of orientations towards the solstices in agreement with the prescriptions of Saint Martin of Braga, who, already in the fifth century, preached against the practice of celebrating the solstice, which apparently was very common among the peoples of the Iberian northwest. However, there are a number of churches that have orientations consistent with solstitial orientations. Particularly striking is the case of San Juan de la Peña (Huesca). This is a Mozarabic church excavated in the rock of an impressive sandstone cliff next to the Serrablo valley. A later Romanesque church was built on top of the Mozarabic one, with a slight change in orientation. The apse of the two churches is directly carved into the cliff and thus there is no horizon. If we consider a flat horizon, the declination is consistent with that of the winter solstice, or perhaps with the celebration of Saint John the Evangelist (27 December). This is one of the few Mozarabic churches to be orientated towards the southern part of the sunrise arch. However, another possibility arises if in this case the opposite direction is considered, facing the cliff towards the other side of the ravine, with an angular height of $2^{\circ}$. Such a direction would have a declination of $24^{\circ}$ and thus be consistent with an orientation towards the summer solstice or the festivity of Saint John the Baptist, precisely the one celebrated in this church.

This opens up the possibility that a number of churches might have been orientated according to sunrise (or sunset in that case) on the patron saint's day. This could indeed be the case for a small number of churches. However, such an investigation would be troublesome to do fairly for all the churches in our sample. The oldest churches are commonly found in archaeological excavations and records of the supposed dedication are absent. In several instances, such dedications 
changed over time, and the original name of the saint or saints celebrated in a particular church is often lost. However, the fact that a statistically significant number of churches of all periods and with varied dedications are orientated towards the east, possibly in coincidence with sunrise on 25 March, would argue against a common use of sunrise on the day of the patron saint.

In spite of this, it is indeed enlightening to perform an interesting exercise with the secondary maxima found in the different periods. The second most prominent concentration of orientations for the Palaeo-Christian churches is that towards 18 April or 25 August. Neither of these dates seems to be close to any major Christian festival, unless we consider 18 April to be close to the date when Easter is commonly celebrated.

The second maximum for Visigoth churches appears close to the dates 21 February and 15 October. It is interesting that on 22 February there is the celebration of the Seat of Saint Peter (Cathedra Petri) a festivity apparently celebrated since the fourth century, commemorating the episcopal office of Saint Peter and the creation of the Church. It is noteworthy that a similar concentration was found in Slovenia for a number of Romanesque churches (Čaval 2009).

Mozarabic churches present a remarkable secondary peak at declination $18.5^{\circ}$, or 9 May and 25 July Julian for A.D. 1050. The first date does not seem to be particularly relevant; in contrast, the second seems more relevant. This is the date for the celebration of Saint James and the most widely commemorated (the other celebrations are on 25 March, his martyrdom, and 30 December, his burial. In comparison to July 25 , these two dates appear to be rather late, as their first mention appears to be in the Codex Calixtinus (Book III, chapter III), a manuscript of the twelfth century). It is significant that this peak appears for the first time in the Mozarabic period as the sepulchre of the Apostle was found in the early ninth century and it was thereafter that the related cult spread while the local kings promoted the cult in Compostela, boosting the creation of the Camino de Santiago. It is significant in this context that an important number of churches built in the north of the Peninsula in the Mozarabic style display an orientation towards sunrise on Saint James's day. Santiago de Peñalba (León) may be mentioned as a particular case. This church is located in the impressive Valle del Silencio, an area known since antiquity for its use as a spiritual retirement for monks and priests. The church was built in the first half of the tenth century as a monastic retreat and houses interesting examples of carvings and paintings. The orientation of the church is such that it should be considered a typical church facing nearly east. However, considering the height of the horizon, the declination is such that it is compatible with the actual sunrise on dates close to Saint James's day.

The churches of Serrablo Valley, although small in number, present quite a characteristic pattern of orientation. With a fairly large group of churches showing orientations compatible with other contemporaneous churches in the Peninsula, perhaps related to sunrise on 25 March, the principal maxima seem to be related to the dates 6 March and 26 September, perhaps associated in this case with the autumnal equinox rather than the spring equinox. This seems to be at odds with the rest of our sample and with the bulk of Medieval churches in Western Europe, but it 
deserves further investigation. One possibility is an influence from the other side of the Pyrenees, as this valley is one of the traditional routes to cross this mountain range, and in this epoch the area was under the nominal rule of the Carolingian kings. Further work in this sense is needed to clarify these issues.

Asturian churches present a particular case of orientations in this study. The main concentration of orientations is towards values of declination $13^{\circ}$, corresponding to 25 April and 18 August Gregorian proleptic, or 20 April and 14-15 August Julian A.D. 830. Only the last of these dates presents a celebration of particularly important interest, as 15 August is the celebration of the Assumption of Mary, although no such festivity was attested in the West until later times. However, as argued earlier, we believe that the Asturian peculiarity would be better expressed as possibly mixing the political or sociological context with liturgical considerations, rather than being a purely religious matter.

\section{Conclusion}

Church alignment in the Iberian Peninsula followed certain specific rules throughout the early Middle Ages. In particular, a vast majority of churches tended to be orientated with the apse facing sunrise on the Vernal equinox, taking this as 25 March, the day of the Annunciation and thus nine months prior to Christmas. This prescription seems to have been followed for almost a thousand years and can be observed in the shift of the main maximum in the orientation histograms through the different time periods. Such a shift is due to the wandering nature of the Julian calendar according to the seasons.

We do not find any compelling evidence that pre-Romanesque churches (see Fig. 10) were orientated towards the point of sunrise on the patron saint's day; however particular dates seem to have been important when deciding the orientation of the churches. The concentration of orientations towards sunrise on 25 July, Saint James's day, found in churches built along the different routes to Santiago, just a few centuries after the discovery of the saint's sepulchre and its promotion as a pilgrimage site, is particularly interesting. These results seem quite robust, given the number of churches measured. However, it would be interesting to test these conclusions. In particular it would be very helpful to do a thorough investigation of the orientation of the early Romanesque churches along the Camino de Santiago in order to analyse whether there was a persistence or a change of the orientation customs of the religious buildings erected in the new style coming from the other side of the Pyrenees.

Acknowledgments Large parts of the fieldwork were carried during these six years of hard work with the help of a series of persons. We would like to especially acknowledge astronomer Lourdes CostaFerrer, anthropologist Margarita Sanz de Lara, and archaeologists Marco García Quintela and Rebeca Blanco Rotea for their ongoing permanent support. This work is partially financed under the framework of the projects AYA2011-26759 "Orientatio ad Sidera III" of the Spanish MINECO, and P310793 "Arqueoastronomía" of the IAC. ACGG is a Ramon y Cajal Fellow of the Spanish MINECO. 


\section{References}

Belmonte, J.A., Tejera Gaspar, A., Perera Betancort, A. and Marrero, R. 2007. On the orientation of preIslamic temples of North Africa: a re-appraisal (new data in Africa Proconsularis). Mediterranean Archaeology and Archaeometry 6 (special issue 3): 77-85.

Chauncey, H. 1700. The Historical Antiquities of Hertfordshire. London. http://books.google.es/book/ about/The_historical_antiquities_of_Hertfordsh.html. Accesed 12 Nov 2014.

Čaval, S. 2009. Astronomical orientations of Sacred Architecture during the Medieval period in Slovenia. In: Cosmology Across Cultures, eds. J.A. Rubiño-Martín, J.A. Belmonte, F. Prada and A. Alberdi, 209-19. San Francisco: Astronomical Society of the Pacific.

Delgado-Gómez, J. 2006. El porqué de la orientación de las iglesias. Lucensia 16 (33): 347-56.

Fontaine, J. 1978a. El prerrománico. Madrid: Encuentro.

Fontaine, J. 1978b. El mozárabe. Madrid: Encuentro.

Esteban, C., Belmonte, J.A., Perera Betancort, M.A., Marrero, R. and Jimenez Gonzalez, J.J. 2001. Orientations of pre-Islamic temples in North-West Africa. Archaeoastronomy 26: S65-84.

Gangui, A., González-García, A.C., Perera Betancort, M.A. and Belmonte J.A. 2015. On the orientation of the historic churches of Lanzarote. In The materiality on the sky, Proc. of the SEAC 2014 Conference, eds. T. Lorsladen and F. Ventura. Bath: Sophia Centre Press, in press.

García Quintela, M.V., González-García, A.C. and Seoane-Veiga, Y. 2014. De los Solsticios en los castros a los santos cristianos; la creación de los paisajes cristianos en Galicia. Madrider Mitteilungen 55: 443-485.

Godoy Fernandez, C. 2004. A los pies del templo. Espacios litúrgicos en contraposición al altar: una revision. Antigüedad Cristiana XXI: 473-89.

González-García, A.C. 2014. A voyage of Christian medieval astronomy: symbolic, ritual and political orientation of churches. In: Stars and Stones, eds. F. Pimenta, N. Ribeiro, F. Silva, N. Campion, A. Joaquinito and L. Tirapicos. British Archaeology Reports: 240-248.

González-García, A.C., Belmonte J.A. 2006. Which Equinox? Archaeoastronomy, The Journal of Astronomy in Culture XX: 97-107.

González-García, A.C., Belmonte J.A. 2014a. Interactions Between Islamic and Christian Traditions in the Iberian Peninsula. In: Handbook of Archaeoastronomy and Ethnoastronomy, ed. C.L.N. Ruggles, 1695-1702. New York: Springer. doi:10.1007/978-1-4614-6141-8_172.

González-García, A.C., Belmonte J.A. 2014b. Sacred architecture orientation across the Mediterranean: a comparative statistical analysis. Mediterranean Archaeology and Archaeometry 14 (2): 95-113.

González-García, A.C., Belmonte J.A. and Costa-Ferrer, L. 2014. The orientation of pre-Romanesque churches in Spain: Asturias, a case of power re-affirmation. In: Astronomy and Power, eds. M.A. Rappenglueck, B. Rappenglueck and N. Campion. British Archaeology Reports, in press.

Johnson, W. 1912. Byways in British Archaeology. Cambridge: Cambridge University Press.

Lockyer, A. 1894. The Dawn of Astronomy. Rpt. Cambridge, MA: MIT Press, 1964.

McCluskey, S.C. 1998. Astronomies and cultures in early Medieval Europe. Cambridge: Cambridge University Press.

McCluskey, S.C. 2004. Astronomy, Time, and Churches in the Early Middle Ages. In: Villard's legacy: Studies in Medieval Technology, Science and Art in Memory of Jean Gimpel, ed. M.-T. Zenner. 197-210. Ashgate: Aldeshot.

McCluskey, S.C. 2010. Calendric cycles, the eighth day of the World and the orientation of English Churches. In: Skywatching in the Ancient World, New Perspectives in Cultural Astronomy, eds. C. Ruggles and G. Urton. 331-353. Boulder: University Press of Colorado.

Nissen, H. 1906. Orientations. Studienzur Geschichte der Religion. Berlin: Weidmann.

Palol i Salellas, P. 1991. Arte y Arqueología, España Visigoda. La monarquía. La cultura. Las artes. Historia de España dirigida por R. Menéndez Pidal, III/2: 269-428. Madrid: Espasa Calpe.

Pérez-Valcarcel, J. 1998. La orientación de las iglesias del Camino de Santiago. In: Actas del Segundo Congreso Nacional de Historia de la Construcción, eds. F. Bores, J. Fernández, S. Huerta, E. Rabasa, 391-396. La Coruña: Servicio de Publicaciones Universidad de La Coruña.

Ruggles, C. L. N. 1999. Whose equinox? Archaeoastronomy 22: S45-50.

Utrero Agudo, M. A. 2006. Iglesias tardoantiguas y altomedievales en la Península Ibérica. Análisis arqueológico y sistemas de abovedamiento. Anejos de Archivo Español de Arqueología, XL. Madrid: Consejo Superior de Investigaciones Científicas. 
Valdivieso Ausín, B. 2006. Ermita de la Virgen del Cerro. Un templo prerrománico en Cueva de Juarros. Burgos: Diputación Provincial de Burgos.

Vogel, C. 1962. Sol aequinoctialis. Problemes et tecnique de l'orientation dans le culture chretien. Revue Sciences Religieuses 36: 175-211.

Wordsworth, W. 1888. The Complete Poetical Works. London: Macmillan and Co.

Antonio César González García (Valladolid, 1973), Ph.D. in Astrophysics (Groningen, The Netherlands) has held postdoctoral fellowships at the IAC (2003-2006 \& 2010-2011) and the Theoretical Physics Department-UAM (2006-2010), where he has investigated in evolution of galaxies and archaeoastronomy (working on the possible astronomical orientation of megalithic monuments in central Europe). Since 2010 he has held a Ramón y Cajal fellowship to work on cultural astronomy of Mediterranean cultures. He is now based at the Instituto de Ciencias del Patrimonio (Incipit-CSIC) at Santiago de Compostela (Galicia, Spain). He is Secretary of the European Society for Astronomy in Culture since 2014. His main lines of research are centred on three issues: modelling of the possible astronomical orientation of classical cultures, possible astronomical and landscape relations of Iron Age sanctuaries and the study of the orientation of ancient Roman cities.

Juan Antonio Belmonte is a staff astronomer at the Instituto de Astrofísica de Canarias (Tenerife, Spain) where he has lectured on the history of astronomy and archaeoastronomy, and investigates in exo planets and cultural astronomy. He has published or edited a dozen books and authored nearly 200 publications on those subjects. He was the Director of the Science and Cosmos Museum of Tenerife from 1995 to 2000 and President of the European Society for Astronomy in Culture (SEAC) from 2005 to 2011. He is Advisory Editor of the Journal for the History of Astronomy. In recent years he has performed extensive research on the astronomical traditions of ancient civilizations, concentrating on the ancient Mediterranean cultures. Born in Murcia (Spain) in 1962, he has been involved in the editing and authorship process of the monumental Handbook of Archaeoastronomy and Ethnoastronomy (Springer, 2014). 